\title{
L'utilisation des groupes bulbes dans les aménagements de basse chute
}

\author{
par P. Cazenave
}

\author{
Directeur honoraire de la Compagnie Nationale du Rhône \\ Président du Comité Scientifique et Technique de la SHF
}

Le 25 septembre 1963, sous la présidence de Pierre Ailleret, je présentais au congrès de Brest de la Société Française des Electriciens une communication intitulée :

«Un exemple d'aménagement de basse chute équipé de groupes bulbes :

Pierre Bénite, sur le Rhône» [1]

En préambule, je soulignais que « les énormes progrès réalisés dans le domaine des gros groupes bulbes industriels étaient dus, pour leur plus grande part, aux études très poussées, aux essais nombreux et à très grande échelle entrepris depuis les années 40 " par les ingénieurs d'EDF en vue de l'utilisation de l'énergie marémotrice, en sorte que Pierre Bénite, « premier grand aménagement de rivière entièrement équipé de groupes bulbes ", pouvait être considéré comme l'enfant de la Rance, même si, par le jeu d'une étrange physiologie, cet « enfant devait naître en même temps que sa mère, voire quelques mois plus tôt ».

De fait, le premier groupe de Pierre Bénite a démarré le 11 mars 1966, celui de la Rance le 19 août de la même année.

«L'étrange physiologie» s'explique par le fait qu'il n'y a pas entre ces deux réalisations une relation de filiation, mais une relation de fraternité.

Les groupes bulbes de la Rance et ceux de Pierre Bénite sont en effet les enfants, pratiquement jumeaux, du groupe de Saint-Malo qui fut la matrice de toute la famille des gros groupes bulbes, riche à l'heure actuelle, en s'en tenant à ceux qui ont une taille comparable ou supérieure à ceux de la Rance, de plus de 300 unités totalisant une puissance de plus de $9000 \mathrm{MW}$.

Je me propose de retracer rapidement l'histoire de cette famille.

\section{I $\square$ LES GROUPES BULBES AVANT SAINT-MALO}

A l'occasion d'une session de la SHF (16-17 novembre 1972) consacrée à «Six ans d'exploitation de l'usine marémotrice de la Rance», j'avais eu la responsabilité d'une séance consacrée aux groupes bulbes ; $\mathrm{j}$ 'avais alors proposé à Monsieur Cotillon de se charger de la partie historique. Il fit, comme je m'y attendais, un travail remarquable [2] auquel je vais beaucoup emprunter dans ce chapitre, et auquel les lecteurs pourront se reporter pour plus de détails.

Les groupes bulbes constituent une branche particulière d'une famille plus large, celle des groupes axiaux.

L'idée de ces groupes axiaux [3] est liée au fait que, lorsque la chute est faible, le parcours moyen de l'eau entre l'amont et l'aval de l'usine devient proche de l'horizontale (fig.1-1)

Il paraît alors barbare, encombrant et générateur de pertes de faire passer le débit dans une bâche spirale qui entoure le groupe et conditionne un entre-axes de groupe très important, puis d'introduire le débit en écoulement centripète dans le distributeur, puis axial vertical dans la roue, pour amorcer finalement un virage de plus de $90^{\circ}$ dans l'aspirateur coudé, qui entraîne un fond de fouilles très profond. Il était donc naturel que plusieurs ingénieurs cherchent à réaliser des machines où l'écoulement resterait axial de l'amont à l'aval, l'axe de rotation de la turbine étant à peu près horizontal.

Le premier brevet en ce sens est celui de la roue à alternateur périphérique de Harza en 1919, complété en 1924 (Fig. 1-2).

D'autres brevets (fig 1-3, 1-4 et 1-5) sont déposés en 1930 (Kühne : roue hélice inclinée avec alternateur extérieur), en août 1933 (Haefele : roue horizontale - multiplicateur à renvoi d'angle - alternateur extérieur à axe vertical), en décembre 1933 (Huguenin : turbine horizontale avec alternateur dans un bulbe amont).

Toutes les idées de principe sont alors déposées. Le problème est de les réaliser.

M. Cotillon explique très bien comment les conditions de l'avant-guerre, puis de la guerre 39-45 donnent à un animateur assez particulier, Arno Fischer, l'occasion d'exploiter ces divers brevets. Curieusement, c'est le groupe bulbe d'Huguenin qui est essayé en premier par une réalisation de deux groupes de $168 \mathrm{~kW}$ installés en Poméranie sur la rivière Persante, à Röstin. C'est un véritable «bulbe amont» avec un alternateur dans une enceinte étanche placée dans le conduit amont (fig. 2). Ces groupes ont fonctionné à puissance réduite (pour des problèmes d'échauffement mal mâ̂- 


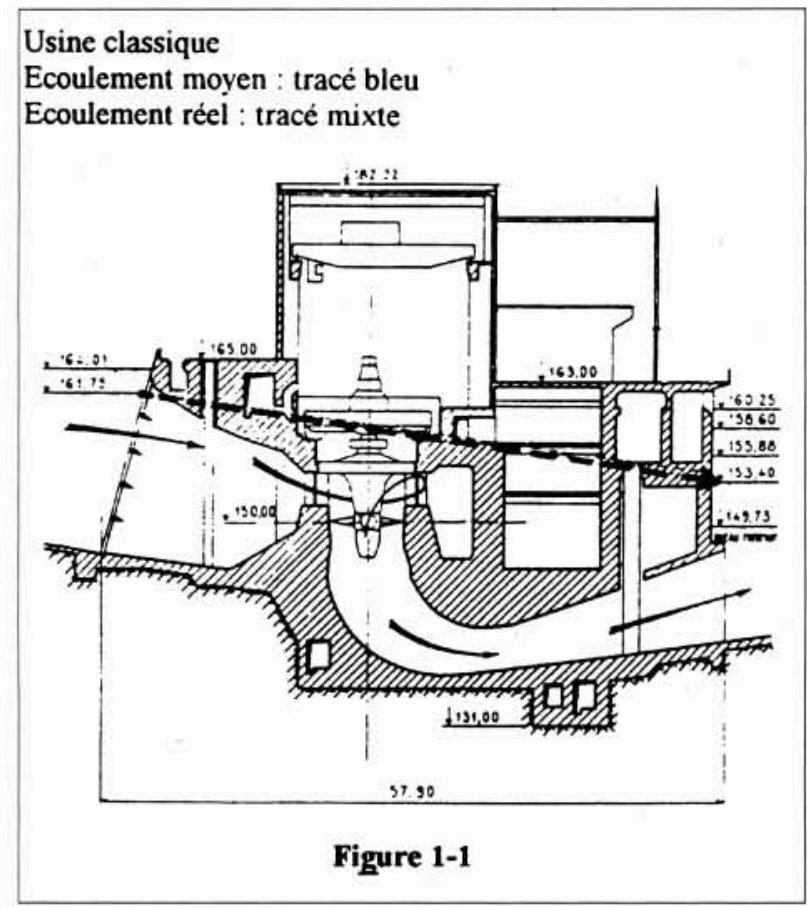

Groupe à alternateur périphérique Brevet Harza 1924

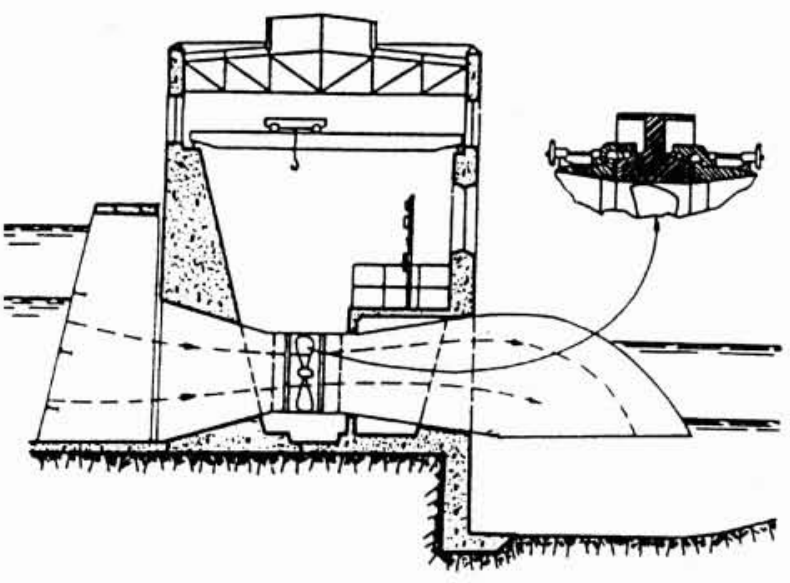

Figure 1-2

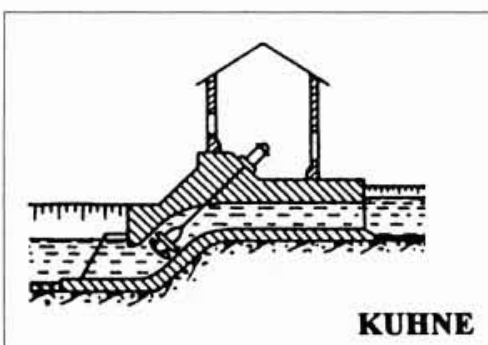

Figure 1-3
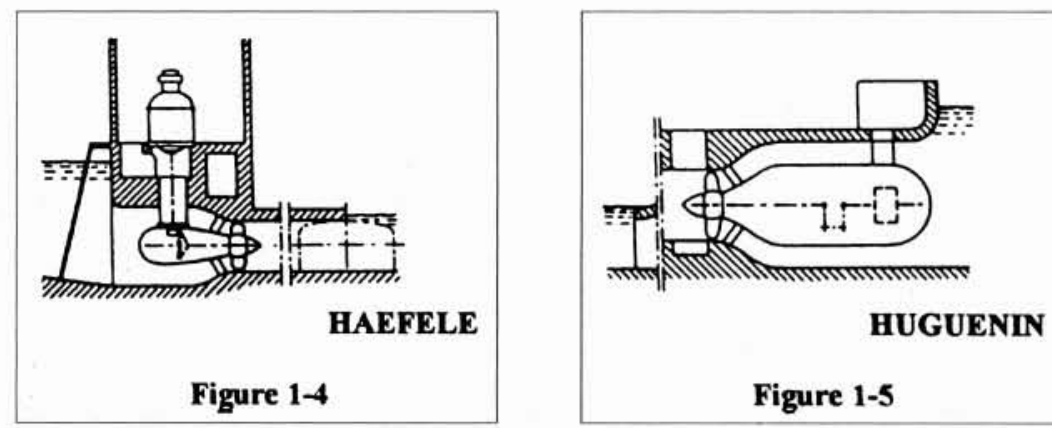

Figure 1-5

1. Usine classique et différents groupes alternateurs bervetés.

trisés) puis ont été abandonnés à la suite de défauts dans le joint d'arbre et de claquages d'alternateurs.

Bien avant ces difficultés, le même Arno Fischer avait lancé tout un programme basé sur des groupes de type Harza à alternateurs périphériques réalisés par Escher Wyss. 73 groupes de ce type, totalisant une centaine de MW, sont installés et en service sur des rivières bavaroises. Ce type de groupes, presque obligatoirement à pales fixes, et présentant d'assez grosses difficultés techniques, a été laissé en sommeil ; mais l'idée en a été reprise sous le nom de Straflow, pour des puissances assez limitées, à l'exception, notable, d'un groupe marémoteur de près de $20 \mathrm{MW}$ à Annapolis.

De même, les solutions à alternateurs extérieurs sont restées limitées à des petites puissances, à l'exception près de deux réalisations américaines (dont une unité de $25 \mathrm{MW}$ ) qui ne paraissent pas avoir eu de suite.

En revanche, le principe des groupes bulbes, issus lointainement de Röstin, est repris sérieusement en France après la guerre, principalement avec l'idée de mettre au point des groupes pour usine marémotrice. Mais la difficulté est qu'en grande puissance, on est condamné à l'entraînement direct de l'alternateur, à la vitesse de la turbine, très faible en rai- son même de la faiblesse de la chute. L'alternateur est donc énorme (fig. 3).

Il faut donc trouver une solution pour réduire l'alésage de la machine, ce qui est impossible avec une ventilation naturelle à l'air. C'est l'ingénieur français Guimbal [4] qui va proposer la première solution viable avec un alternateur tournant dans l'huile, qui sera réalisé pour une usine de la SNCF à Castet en 1954 (fig. 4) (2 groupes de 800 kW).

Cette technique de l'alternateur dans l'huile s'est cantonnée par la suite dans de petites puissances, mais il est incontestable que Guimbal avait montré une voie, et remonté le moral des ingénieurs, qui trouveront peu après la solution de la réfrigération à l'air surpressé, qui s'est révélée tout à fait féconde.

EDF a alors réalisé deux usines laboratoires dans le Massif Central, à Cambeyrac et à Argentat, avec des groupes de techniques diverses, dont en particulier des groupes à «bulbe aval» (alternateur en bulbe dans l'aspirateur) et finalement c'est la solution bulbe amont qui est sortie victorieuse des essais et des comparaisons.

A partir de là, deux équipes EDF vont s'attaquer, l'une à la réalisation d'un groupe plus important pour étude «en 


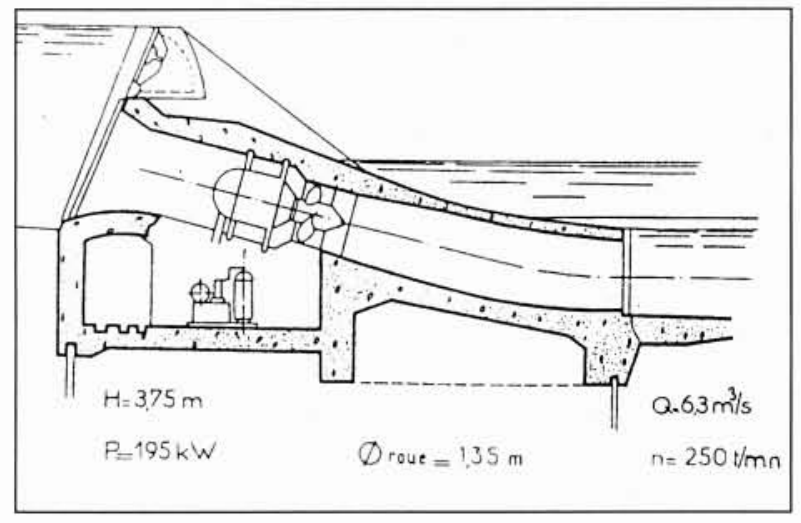

2. Groupe de Rostin. Rivière Persante (Poméranie). 2 groupes de $168 \mathrm{~kW}$. Fonctionnement à environ $110 \mathrm{~kW}$ de 1936 à 1944.

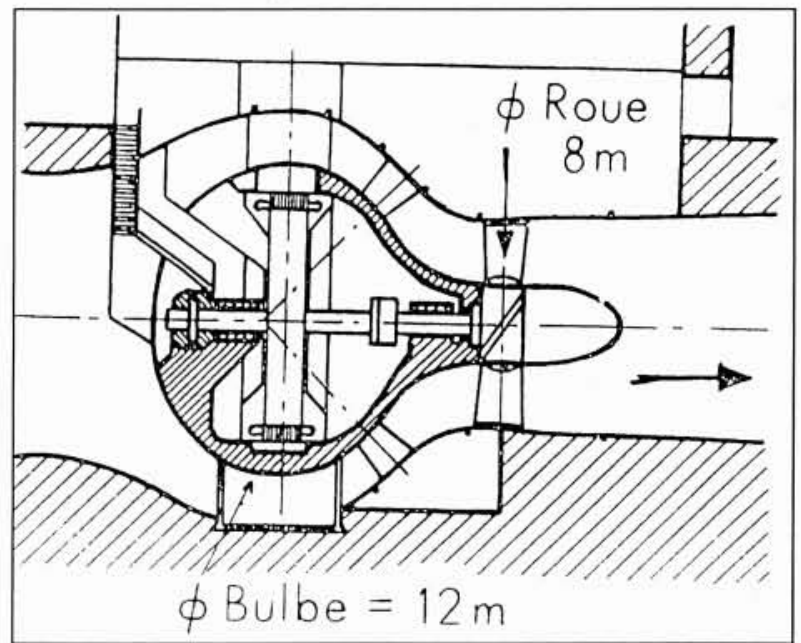

3. Le premier projet de groupe bulbe pour la Rance (1943).

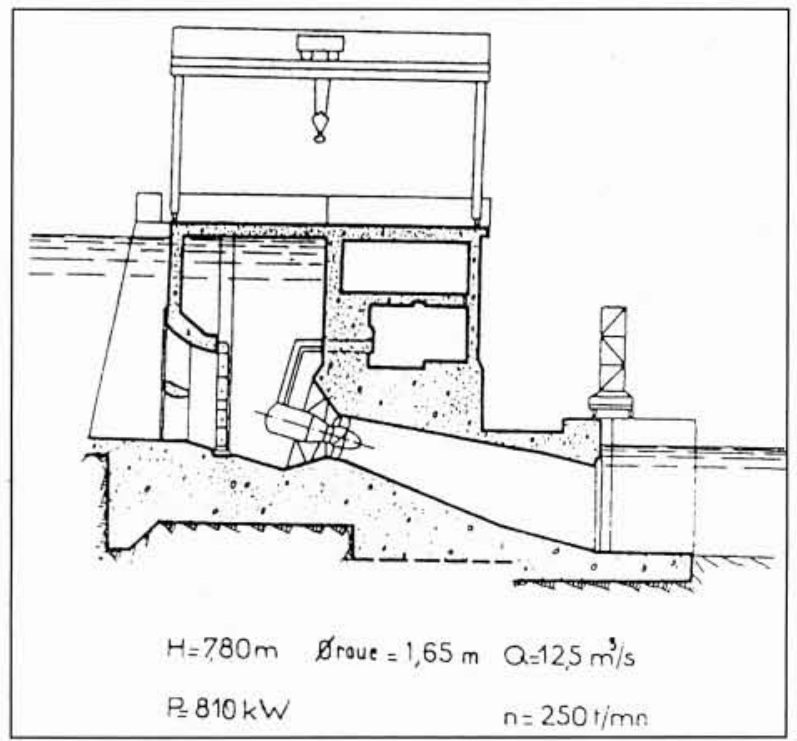

4. CASTET. Gave d'Ossau. Deux groupes de $810 \mathrm{~kW}$. Réfrigération à l'huile (procédé Guimbal). rivière» à Beaumont-Monteux (roue de $3,80 \mathrm{~m}$ de diamètre tournant à $150 \mathrm{tr} / \mathrm{mn}$, alternateur refroidi à l'air à pression atmosphérique), l'autre à un groupe expérimental en milieu marin, avec tous les types de fonctionnements d'usine marémotrice, installé dans l'écluse de Saint-Malo. Ce groupe, maintenant démonté, avait une roue de $5,80 \mathrm{~m}$ de diamètre, tournant à $88,3 \mathrm{tr} / \mathrm{mn}$ et une puissance de $9 \mathrm{MW}$. Les deux installations ont été mises en service en 1959.

C'est sur l'expérience de ces deux groupes, et particulièrement sur l'extrapolation de Saint-Malo, que sera basé le projet de Pierre Bénite. L'organigramme de la figure 5 schématise cette filiation, et la fraternité avec la Rance.

Et, pour leur rendre l'hommage qu'ils méritent, j'ai porté sur ce schéma les noms des principaux ingénieurs responsables que j'ai rencontrés au cours de ces études.

\section{II — DE SAINT-MALO À PIERRE BÉNITE}

Les études de l'aménagement hydroélectrique de Pierre Bénite sur le Rhône ont commencé dans les années 1960. Le choix du site, juste en aval de Lyon, était dû principalement à des objectifs de navigation (liaison entre Rhône et Saône) et ne permettait d'utiliser qu'une chute modeste. En outre, l'essentiel de la chute étant obtenu "par l'aval» à l'aide d'un long canal de fuite, la chute varie beaucoup avec le débit (11 $\mathrm{m}$ à l'étiage, $8 \mathrm{~m}$ au point de puissance maximale, $5 \mathrm{~m}$ au débit de 10 jours).

En solution Kaplan traditionnelle, l'équipement optimal était de 4 groupes à roues de $7 \mathrm{~m}$ de diamètre tournant à $71,4 \mathrm{tr} / \mathrm{mn}$, avec un entre-axes de $24 \mathrm{~m}$.

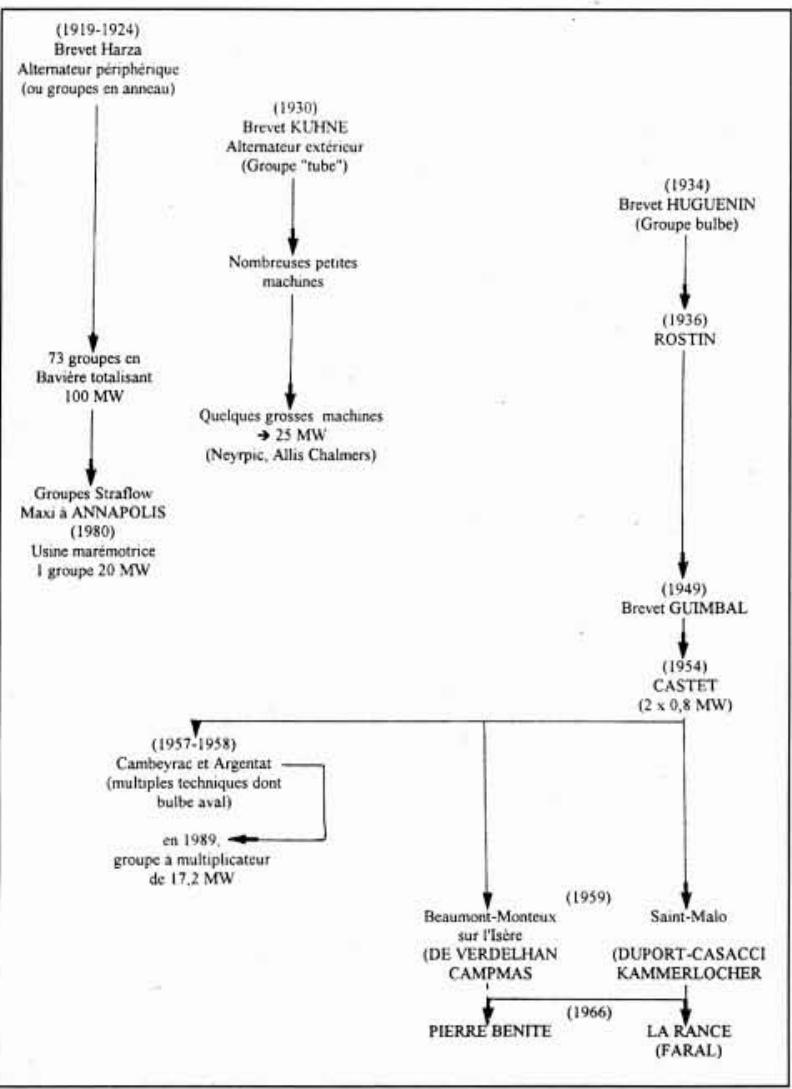

5. Genèse des groupes de la Rance et de Pierre Bénite. 


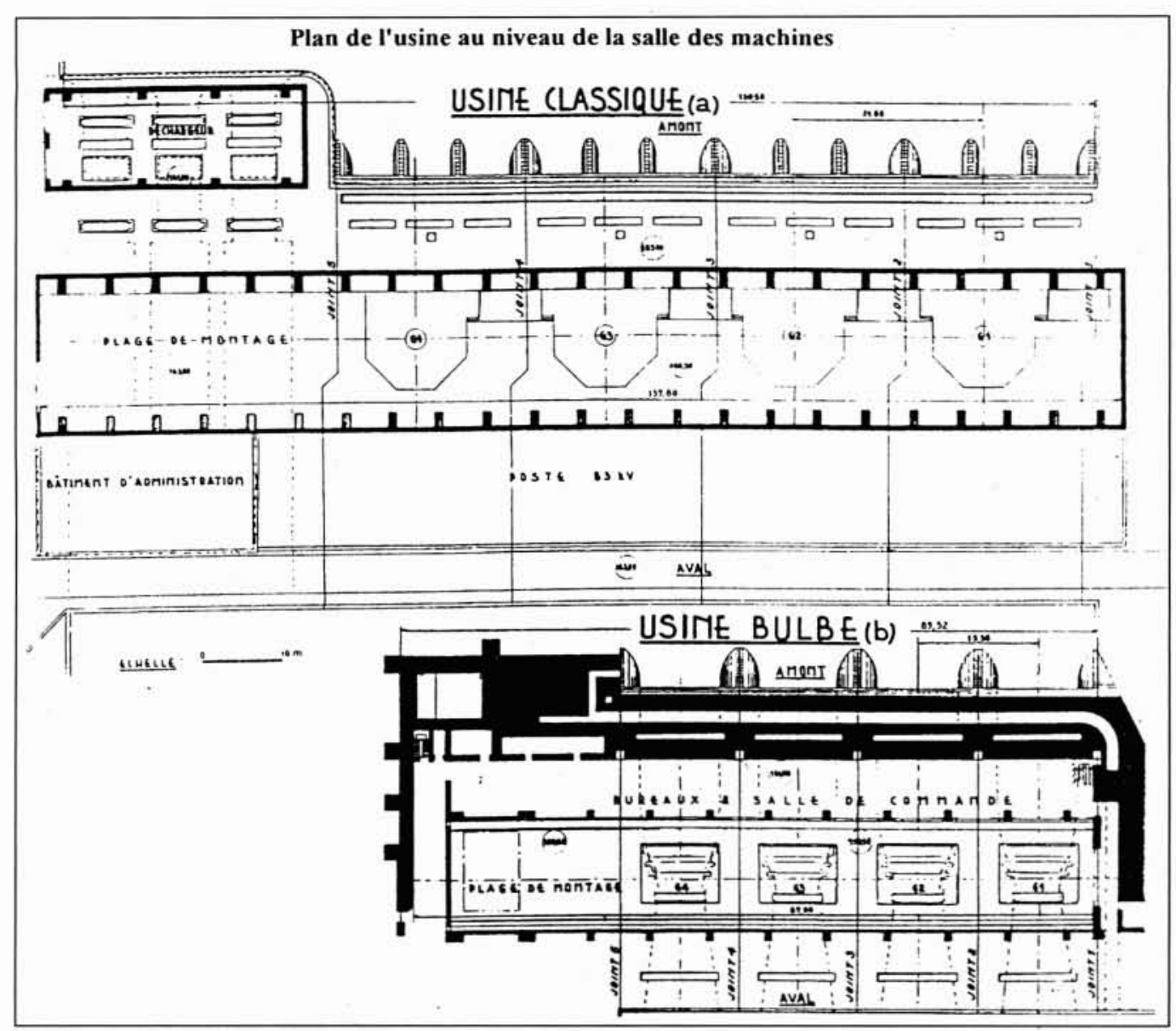

L'étude préalable montrait que la solution à groupes bulbes permettait une réduction du génie civil très importante, en coupe, et surtout en plan, où on note (fig. 6) une diminution de plus de $30 \%$ sur la dimension en travers du fleuve.

La décision était cependant difficile à prendre, comme le soulignait Pierre Ailleret, en ces termes en ouvrant la discussion au congrès de la S.F.E. : C'est à la Compagnie Nationale du Rhône que revient le mérite d'avoir ouvert l'ère vraiment industrielle du groupe bulbe car, après tout, à la Rance, nous n'avions guère le choix qu'entre employer des groupes bulbes, ou ne rien faire du tout. C'est la première fois à Pierre Benite que s'est posé le problème de situer la centrale à groupes bulbes par rapport à l'aménagement en groupes conventionnels... Je crois que c'est vraiment de Pierre Bénite que commence la période des groupes bulbes, que nous allons voir se poursuivre à Gerstheim et sur d'autres «aménagements...».

Il y a dans ces phrases un résumé des hésitations de l'époque. Si les hommes de l'équipement étaient très favorables à cette innovation, ceux de l'exploitation avaient naturellement une certaine inquiétude.

En outre, au stade préliminaire auquel je faisais allusion tout à l'heure, s'il était sûr qu'on gagnerait sur le génie civil, on n'était pas certain que ces machines nouvelles, à mécanisme difficile (roue en porte à faux impliquant une forte charge sur le palier, distributeur conique, montage à l'horizontale...) et surtout à conception électrique poussée (fortes densités de courant, évacuation des pertes par ventilation forcée, faible longueur des pas polaires...) ne conduisent pas à un coût plus élevé des machines. Et il ne faut pas oublier que dans une usine de basse chute, l'équipement représente $2 / 3$ du coût, contre seulement $1 / 3$ pour le génie civil. Il fallait donc procéder à une étude complète des machines avant de se décider.

Pour passer de Saint-Malo à Pierre Bénite, on ne s'est pas contenté de simples extrapolations ; on a "épuré» la technique de toutes les technologies coûteuses liées à l'eau de mer, on a repensé tous les systèmes de réfrigération, réexaminé tous les systèmes de montage, et l'accessibilité du personnel. Lorsque nous avions, avec Monsieur Henry, Directeur des Etudes, visité en détail Saint-Malo, sous l'éminente conduite de Messieurs Mauboussin et Kammerlocher, nous étions descendus dans le bulbe par des échelles. J'étais jeune, Monsieur Henry l'était moins, et il s'était justement inquiété des fatigues liées à ces sortes d'accès. En raison de cette inquiétude, et pour donner aux exploitants futurs un confort voisin de celui qu'ils connaissaient dans les usines classiques, j'avais fait étudier par Neyrpic une galerie horizontale réunissant tous les bulbes en leur partie amont. Cette solution (fig. 7) qui, selon l'expression de Monsieur Faral, «débulbise» les groupes bulbes, avait en outre l'avantage de constituer un appui mécanique essentiel (qui permettra plus tard, dans la série Vallabrègues, un allègement notable de l'avant-distributeur), et d'offrir une importante surface d'échange qui nous permettait un système de réfrigération très efficace.

Ma communication de Brest [1] se situe après toutes ces études, et après l'engagement de prix ferme des constructeurs. Elle montre alors qu'à l'économie de génie civil, confirmée, vient s'ajouter une économie de $15 \%$ sur l'équipement, une très forte réduction de l'ouvrage déchargeur, et 


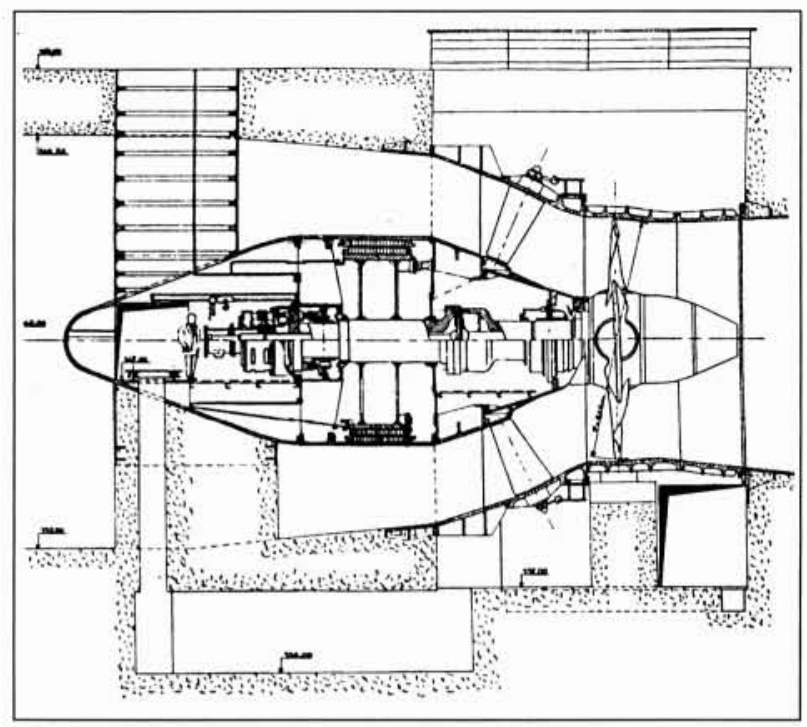

7. Ensemble du groupe de Pierre Bénite.

au total l'économie est de l'ordre de $20 \%$ de la totalité de l'ouvrage usine.

Cela tient en particulier au fait que la roue bulbe «équivalente» est sensiblement plus petite que la roue Kaplan. La communication de Brest [1] déjà citée montre que les collines d'une roue classique de $7 \mathrm{~m}$ tournant à $71,4 \mathrm{tr} / \mathrm{mn}$ et d'une bonne roue bulbe, spécifiquement tracée dans ce cadre, et pour un emploi essentiellement limité au turbinage direct, ayant un diamètre de $6,10 \mathrm{~m}$ tournant à $83,3 \mathrm{tr} / \mathrm{mn}$, coïncident pratiquement dans tout le domaine d'exploitation (fig. 8)

$$
\text { Le rapport } \frac{7}{6,1} \cong \frac{83,3}{71,4} \cong 1,15
$$

a été souvent cité par la suite comme rapport «d'équivalence» entre Kaplan classique et bulbe.

De ce fait, le poids de la turbine bulbe devient sensiblement inférieur au poids de la turbine Kaplan ; cet allègement vient s'ajouter à celui, naturel, de l'alternateur dont les dimensions sont beaucoup plus faibles que celles de l'alternateur traditionnel, si bien que le poids total du groupe est réduit de $27 \%$, comme le montre le tableau 1 .

Tableau 1 : Poids des groupes «équivalents» (pour un groupe).

\begin{tabular}{|l|c|c|c|}
\hline & $\begin{array}{c}\text { Groupe } \\
\text { traditionnel }\end{array}$ & $\begin{array}{c}\text { Groupe } \\
\text { bulbe }\end{array}$ & $\begin{array}{c}\text { Gain de } \\
\text { poids }\end{array}$ \\
\hline Turbine & $720 \mathrm{t}$ & $757 \mathrm{t}$ & $145 \mathrm{t}(20 \%)$ \\
Alternateur & $270 \mathrm{t}$ & $145 \mathrm{t}$ & $125 \mathrm{t}(46 \%)$ \\
Groupe complet & $990 \mathrm{t}$ & $720 \mathrm{t}$ & $270 \mathrm{t}(27 \%)$ \\
\hline
\end{tabular}

Dès lors, $\mathrm{j}$ 'avais le feu vert de ma direction générale. Celui d'EDF me fut donné par Monsieur Wyart dont j'ai, en d'autres lieux, souligné le grand souci de l'intérêt général, et il restait à convaincre les échelons locaux qui allaient recevoir cet objet nouveau : Monsieur Anglaret, alors chef du GRPH Rhône, Monsieur Revol, chef du centre de transport, Monsieur Hilaire, Chef du Centre de Mouvement d'Energie,

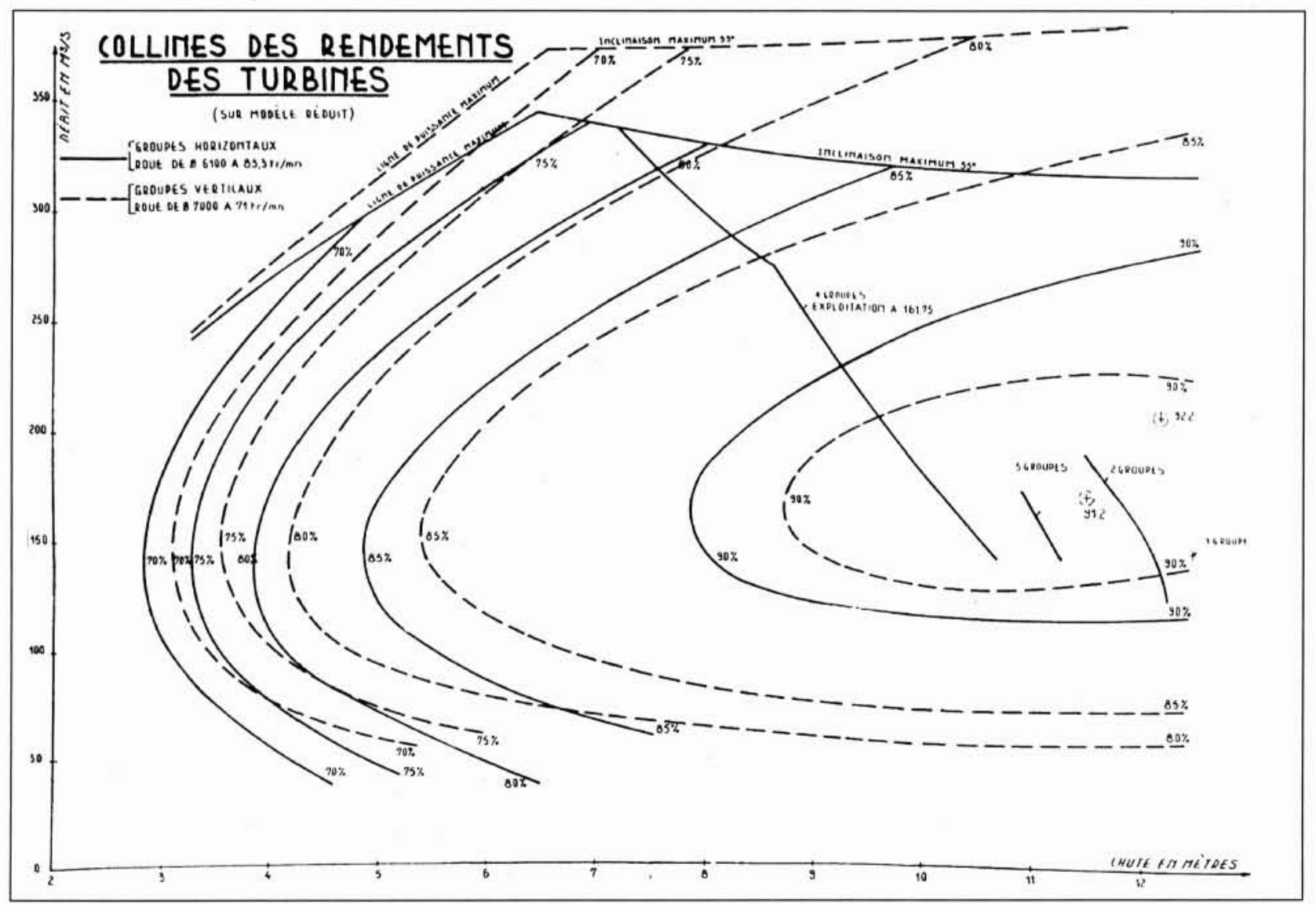

8. Collines des rendements de turbines. 
Tableau 2 Gros groupes bulbes (20 MW et plus en rivière, $10 \mathrm{MW}$ et plus en usines marémotrices). Situation par pays d'installations.

\begin{tabular}{|l|c|c|c|c|}
\hline \multicolumn{1}{|c|}{$\begin{array}{c}\text { Pays des } \\
\text { installations }\end{array}$} & $\begin{array}{c}\text { Puissance } \\
\text { totale } \\
\text { en MW }\end{array}$ & $\begin{array}{c}\text { Nombre } \\
\text { de } \\
\text { groupes }\end{array}$ & \multicolumn{2}{|c|}{ Plus gros groupe } \\
Puissances & Site \\
\hline France & 2027 & 83 & 46,5 & Chautagne \\
Autriche & 1640 & 58 & 48,4 & Ybbs \\
USA & 1482 & 50 & 53,5 & Rock Island \\
Chine & 765 & 28 & 31 & Lingintan \\
Ukraine & 647 & 26 & 23 & Kanev \\
ex-Yougoslavie & 392 & 12 & 41,7 & Dubrava \\
Canada & 224 & 8 & 29 & Jenpeg \\
Japon & 188 & 5 & 66 & Tadami \\
Roumanie & 168 & 6 & 29 & Portes de Fer \\
Russie & 166 & 8 & 47,5 & Saratov \\
Portugal & 164 & 4 & 43 & Crestuma \\
Suède & 154 & 6 & 32 & Torron \\
Thaïlande & 142 & 4 & 35,4 & Pak Man \\
Pakistan & 132 & 6 & 22 & Chasma \\
Corée & 127 & 4 & 31,7 & Paldang \\
& & & & réhabilitation) \\
Brésil & 127 & 4 & 44 & Igarapava \\
Allemagne & 108 & 4 & 27 & Iffezheim \\
Norvège & 99 & 4 & 36,8 & Haugsdal \\
Finlande & 94 & 4 & 27,4 & Kurkiaska \\
Irak & 80 & 4 & 20 & Mosul \\
\hline
\end{tabular}

et le regretté Monsieur Ginouze, Ingénieur en chef à la Distribution, car cette centrale allait faire un apport assez massif sur un réseau local très industrialisé. Nous avions décidé ensemble de tenter cette expérience intéressante, et de la tester ultérieurement en vraie grandeur par un essai «d'îlotage», qui eut lieu en 1967, avec trois groupes ilotés sur un réseau industriel de $30 \mathrm{MW}$, ayant les caractéristiques statistiques de variabilité comparables à celles du réseau européen [5]. Le résultat satisfaisant de ces essais élimina les réticences liées aux problèmes de régulation, et dédouana l'expansion à venir dont nous parlerons plus loin.

Compte tenu de tous ces avis, la décision d'équipement en groupes bulbes fut prise en 1962. Un an plus tard, EDF commandait à son tour 6 groupes bulbes, un peu plus puissants (24 MW) - mais, compte tenu d'une chute plus favorable, de plus petit diamètre $(5,60 \mathrm{~m})$-pour équiper l'aménagement de Gerstheim sur le Rhin.

\section{DE PIERRE BÉNITE À 1996}

Comme on vient de le voir, Pierre Bénite a ouvert la voie aux gros groupes bulbes industriels.

Pierre Bénite était le 7ème aménagement du Rhône. Il en restait 11 à équiper, tous de basse chute

Tableau 3. Gros groupes bulbes ( $20 \mathrm{MW}$ et plus en rivière, $10 \mathrm{MW}$ et plus en usines marémotrices) en service ou en construction en 1996.

\begin{tabular}{|c|c|c|c|c|c|}
\hline \multicolumn{2}{|c|}{ Constructeurs } & \multirow{2}{*}{$\begin{array}{c}\text { Puissance } \\
\text { totale } \\
\text { (MW) }\end{array}$} & \multirow{2}{*}{$\begin{array}{c}\text { Nombre } \\
\text { de } \\
\text { groupes }\end{array}$} & \multicolumn{2}{|r|}{ Plus gros groupes } \\
\hline Nom & Pays & & & Puissance & Site \\
\hline NEYRPIC & France & 2902 & 104 & 53,5 & ROCK ISLAND (USA \\
\hline SULZER-ESCHER-WYSS & Suisse & 881 & 30 & 39 & ALTENWORTH (Autriche) \\
\hline VOEST ALPINE & Autriche & 855 & 27 & 44 & IGARAPAVA (Brésil) \\
\hline KTZ & Ukraine & 647 & 26 & 47,5 & SARATOV (Russie) \\
\hline VOITH & Allemagne & 591 & 28 & 40,6 & SHINGOH (Japon) \\
\hline LMZ & Russie & 564 & 22 & 29 & IGARAPAVA (Brésil) \\
\hline FUJI & Japon & 477 & 18 & 40,6 & SHINGOH (Japon) \\
\hline ANDRITZ & Autriche & 372 & 13 & 48,4 & YBBS (Autriche) \\
\hline ALLIS-CHALMERS & USA & 236 & 10 & 25 & OZARK LOCK (USA) \\
\hline NOHAB & Suède & 220 & 8 & 39 & ALTENWORTH (Autriche) \\
\hline HITACHI & Japon & 212 & 6 & 66 & TADAMI (Japon) \\
\hline BOVING & Grande-Bretagne & 200 & 8 & 25 & SIDNEY-MURRAY (USA) \\
\hline ROMENERGO & Roumanie & 168 & 6 & 28 & PORTES DE FER (Roumanie) \\
\hline HARBIN & Chine & 164 & 6 & 31 & LINGJINTAN (Chine) \\
\hline KVAERNER & Norvège & 154 & 6 & 36,8 & HAUGSDAL (Norvège) \\
\hline KMW & Suède & 154 & 6 & 31,6 & TORRON (Suède) \\
\hline LITOSTROJ & ex-Yougoslavie & 83 & 2 & 41,7 & DUBRAVA (ex-Yougoslavie) \\
\hline TAMPELLA & Finlande & 46 & 2 & 24,3 & KOKKOSNIVA (Finlande) \\
\hline
\end{tabular}


et appelant en général des groupes de l'ordre de $30 \mathrm{MW}$ do puissance unitaire, ce gui engageait à une nowvelle extrapolation.

Chronologiquement, le premier aménagement ì construire ensurte était celur de Bourg-les-Valence, dont la mise en service étail préwue au débul de 1968, ce qui impliquait de démarrer la construction des machines at début de 1965 , done 15 mois avant le démarage des múchines de PiereBénite. Il n'étajt donc pas possible de bénéficier de l'expérience de Pierre Bénite pour en tenir compte dans la conception des groupes de Bourg-les-Valence. Ceux-ci furent donc commandés en conception traditionnelle (Kaplan verticale). Par contre, Vallebrègues qui venail après, devait dématrer en avril 1970, ce qui conduisait à une commande des groupes en 1967; il serait donc possible de tenir comple de l'expérience de Pierre Bénite pour corriger éventuchement certains éléments de la conception de Vallabrègues. Ceux-ci furent donc commandés en 6 groupes bulbes de 6,25 m cle diamètre de rote, pour unc puissance unitaire de $35 \mathrm{MW}$; at toutes les usines suivantes furent équipées en bulbes, allant jusqu'ì des puissances unitaires de 46,5 MW soit au total, sur le Rhône, en 1986, onze aménagements équipés de groupes bulbes, au nombre de 42 , totalisant une puissance de $1327 \mathrm{MW}$.

De son côté, comme on l'a vu, EDF décidait d'équiper de groupes bulbes les trois centrales du Rhin à partir de Gerstheim (Gerstheim. Strasbourg. Gambshein) soit au total 26 groupes totalisant 413 MW (auxquels s'ajoutent les 4 groupes d'Ifferheim en Allemagne totalisant $108 \mathrm{MW}$ ), et les 3 groupes de Golfesh, totalisant $70 \mathrm{MW}$, sur la Gatonne.

L'Autriche ouvre dès 1969 un gros programme d'équipement du Darube en groupes bulbes, et les deux constructeurs d'URSS mettent en construction ì partir de 1962 un grand programme sur les heuves d'Ukraine et de Russie, en sortc qu'en 1986, lorsque le programme du Rhône s'achc̀ve, la puissance totale des gros groupes bulbes (c'est-à-dire les groupes de $20 \mathrm{MW}$ et plus cn rivière et les groupes do $10 \mathrm{MW}$ et plus en usines maremotrices) instatlés dans le monde approchait $7000 \mathrm{MW}$, A la fin de 1996, ce total atteint $9000 \mathrm{MW}$ en plus de 300 groupes, avec des puissarces unitaires avoisinant couramment $50 \mathrm{MW}$; un groupe mis cn scrvice en 1990 (Tadami) atteint même $66 \mathrm{MW}$.

Les tableaux 2 et 3 montrent comment se répartissent ces groupes aujourd'hui. Ils mettent en évidence que les écuipements, partis des grands fleuves d'Europe occidentale (Danube, Rhône, Rhin) et de l'ex-LRSS (principalement Russie et Ukraine) ont très tôt traversé l'Atlantique (Rock Isiand, avec 8 groupes de 53,5 MW date de 1974) et a gragné l'Asie, notamment la Coréc dès 1966, lc Japon à partir de I975 et massivement la Chine à partir de 1978 .

\section{IV - PERSPECTIVES D'AVENIR}

Le tableau 4 résume les intentions d'équipement des divers pays, connues en 1996. On relève un total d'environ 270 groupes totalisant une nouvelle tranche de puissance de près
Tabfeau 4 Groupes bulbes de $20 \mathrm{MW}$ ct plus. Intentions d'équipement connues en 1996

\begin{tabular}{|l|c|c|c|}
\hline \multicolumn{1}{|c|}{ Sites } & $\begin{array}{c}\text { Puissance } \\
\text { totale } \\
\text { (MW) }\end{array}$ & $\begin{array}{c}\text { Aombre } \\
\text { de } \\
\text { groupes }\end{array}$ & $\begin{array}{c}\text { Puissance } \\
\text { unitaire } \\
\text { maximale }\end{array}$ \\
\hline Chine (42) & 6664 & 204 & 50 \\
\hline Inde (3) & 1112 & 41 & 37 \\
\hline Canada (2) & 310 & 5 & 75 \\
\hline Brésil (3) & 201 & 8 & 28 \\
\hline Allemagne (2) & 155 & 5 & 32 \\
\hline USA (1) & 90 & 3 & 30 \\
\hline Corée (1) & 8 & 4 & 21 \\
\hline Azerbaidjan (1) & 39 & 1 & 39 \\
\hline
\end{tabular}

de $9000 \mathrm{MW}$. Si on fait l'hypothèse que ce total représente une perspective à l'horizon de 10 ì 15 ans, on peut prévoir une puissance totale des gros groupes bulbes de l'ordre de $20000 \mathrm{MW}$ en: 2010 , sans compter les usines maréfnotrices actuellement en gestation.

La petite graine de 10.MW plantée dans l'eau salée de l'écluse de Saint-Malo en vue du prestigieux projet de la Rance, et arrosće par l'eau douce et abondante du Rhône à Pierre Bénite, a donné naissance à un arbre mondial, qui a encore de nombreux bourgeons!

\section{REFERENCES}

[1] P. CAZENAVE - CNR - «Ln exemple d'aménagement de basse chute Équipé de groupes bulbes - PIERRE BENITE, sur te Rhône» - Comple rendu du congrès de Brest de la Société Française des Electriciens (25 septembre 1963) er Revue Généralc de l'Electricité Novembre 1964.

[2] J. COTILLON - EDF - «Les Groupes Bulbes : de Röstin en Avignon, l'cssor d'une technique» - La Hourlle Blanche 2/3 - 1973.

[3] P. CAZENAVE - CNR - «L'usine hydroélectrique de Pierte Bénite» - Revue Travaux - Octobre-Novembre 1967.

[4] H. CHAMAYOU et J. GUIMBAI - «Les nouveaux groupes de la centrale protolype de CASTET» - La Houlle Blanche $n^{\circ} 2$ - Mars-Avril 1954.

65: P. BOLSSLGES, P. CAMPMAS, P. CAZENAVE, J. CHEVALIER, M. LECRIQLE - «La régulation de vitesse des groupes bulbes de forte puissance (voir en particulier titre 2-1) - SHF 9èmes Journées de l'Hydraulique - Paris 1966.

\section{N. B. Chacun des documents cités renvoic à de nombreuses autres références}

